\title{
Determinação da incidência, dos níveis de gravidade e da realidade do Transtorno do Espectro do Autismo (TEA) em usuários de instituições de apoio de um município do Amazonas
}

\author{
Determining the incidence, severity levels and reality of Autism Spectrum Disorder (ASD) \\ in users of support institutions in a municipality in Amazonas
}

Determinar la incidencia, niveles de severidad y realidad del Trastorno del Espectro

Autista (TEA) en usuarios de instituciones de apoyo en un municipio de Amazonas

Walter de Jesús García-Parra ${ }^{1}$, Jaida Souza da Costa ${ }^{1}$, Flavia Decaris Rolim ${ }^{1 *}$.

\begin{abstract}
RESUMO
Objetivo: Determinar a incidência, os níveis de gravidade, a frequência, evidenciar as ações diagnósticas realizadas pelos profissionais, compreender e identificar o processo de acompanhamento psicológico de instituições de apoio a pessoas com Transtorno do Espectro do Autismo (TEA) de um município do Amazonas. Métodos: Aplicou-se o instrumento Childhood Autism Rating Scale (CARS) com os autistas diagnosticados, e foi construído pela aluna três tipos de entrevistas especificas a cada categoria, aos pais, aos psicólogos e aos médicos, visando entender melhor as dificuldades e condutas. O estudo foi aprovado por Comitê de Ética em Pesquisa. Resultados: Correspondem a 118 crianças e responsáveis, concluindo-se que 46 crianças possuem TEA severo, 19 moderado, 36 leve, sendo 4 Asperger e 13 não estavam dentro do TEA, além da maioria das crianças fazerem uso de medicação de uso controlado e terem alguma alergia alimentar. Conclusão: Com isso, observou-se que o envolvimento da família é fundamental para o progresso da criança, assim como o acompanhamento de uma equipe multidisciplinar.
\end{abstract}

Palavras-chave: Atendimento multidisciplinar, Autismo, CARS, Neurodesenvolvimento, Interação social.

\section{ABSTRACT}

Objective: To determine the incidence, severity levels, frequency, highlight the diagnostic actions performed by professionals, understand and identify the process of psychological monitoring of support institutions for people with Autism Spectrum Disorder (ASD) in a municipality in Amazonas. Methods: The Childhood Autism Rating Scale (CARS) instrument was applied to diagnosed autistics, and the student constructed three types of specific interviews for each category, with parents, psychologists and physicians, in order to better understand the difficulties and behaviors. The study was approved by the Research Ethics Committee. Results: They correspond to 118 children and guardians, concluding that 46 children have severe ASD, 19 moderate, 36 mild, 4 Asperger's and 13 were not in the ASD, in addition to the fact that most children use controlled medication and have any food allergies. Conclusion: With this, it was observed that the involvement of the family is essential for the child's progress, as well as the monitoring of a multidisciplinary team.

Keywords: Multidisciplinary care, Autism, CARS, Neurodevelopment, Social interaction.

\section{RESUMEN}

Objetivo: Determinar la incidencia, niveles de severidad, frecuencia, resaltar las acciones diagnósticas realizadas por los profesionales, comprender e identificar el proceso de seguimiento psicológico de las instituciones de apoyo a personas con Trastorno del Espectro Autista (TEA) en un municipio de Amazonas. Métodos: Se aplicó el instrumento Childhood Autism Rating Scale (CARS) a los autistas diagnosticados, y el estudiante construyó tres tipos de entrevistas específicas para cada categoría, con padres, psicólogos y médicos, con el fin de comprender mejor las dificultades y conductas. El estudio fue aprobado por el Comité de Ética en Investigación. Resultados: Corresponden a 118 niños y tutores, concluyendo que 46 niños tienen TEA severo, 19 moderados, 36 leves, 4 Asperger y 13 no estaban en TEA, además de que la mayoría de los niños usan medicación controlada y tienen alguna alergia alimentaria. Conclusión: Con esto, se observó que la participación de la familia es fundamental para el progreso del niño, así como el seguimiento de un equipo multidisciplinario.

Palabras clave: Atención multidisciplinar, Autismo, CARS, Neurodesarrollo, Interacción social.

1 Universidade Nilton Lins, Manaus - AM. *E-mail: flaviadecaris_95@hotmail.com

SUBMETIDO EM: 11/2021

ACEITO EM: 11/2021

PUBLICADO EM: 11/2021 


\section{INTRODUÇÃO}

Segundo Sousa PML e Santos IMSC (2018) o Transtorno do Espectro Autista (TEA) é um distúrbio do neurodesenvolvimento que tem como caracteristicas prejuízos nas áreas sociocomunicativas e comportamentais, influenciando em diferentes níveis na comunicação de alunos autistas, ele pode ser associado a outras comorbidades como deficiência intelectual, dificuldades de coordenação motora e de atenção, hiperatividade, dislexia, dispraxia, transtorno depressivo e de ansiedade e, as vezes problemas com o sono, alimentação e distúrbios gastrointestinais (DIAGNOSTIC AND STATISTICAL MANUAL OF MENTAL DISORDERS (DSM-5), 2013).

Indivíduos classificados no nível leve do TEA necessitam de suporte espontâneo, apresentando dificuldade de comunicação, reduzida vontade de se socializar e uma fixação nos seus interesses que são restritos. $\mathrm{Na}$ classificação moderada há necessidade de substancial suporte, possuindo um grau maior de dificuldade em comunicação verbal e não verbal, precisando de ajuda para interagir e responder ao ambiente, além de apresentarem muita frustração ao mudar de rotinas e protocolos habituais. Os de classificação severa necessitam de suporte intenso e substancial, há severo déficit de comunicação, interação muito limitada e grave reação às mudanças (ALMEDA CM e ALBUQUERQUE K, 2017).

A síndrome de Asperger é considerada pelo Manual Diagnóstico e Estatístico de Transtornos Mentais (DSM-5) um transtorno neurobiológico enquadrado como TEA nível 1. É caracterizada por dificuldades de relacionamento interpessoal e comportamento "autístico", mas com bom nível cognitivo, comunicação e interação social (DSM-5, 2013).

De acordo com Almeda CM e Albuquerque K (2017) o diagnóstico do autismo é clínico, para isso, é importante a união dos profissionais que acompanham a criança. De acordo com Riesgo R, et al. (2015) como - TEA é um transtorno de comportamento, os profissionais da área de saúde, educadores e/ou acompanhantes das pessoas com suspeita do transtorno podem utilizar algumas escalas, algumas com livre acesso ao público, para diagnosticar.

Pereira JRS, et al. (2021) afirma que nos últimos anos começou-se a debater mais sobre o TEA, porém ainda falta muito para termos um conhecimento expansivo, diante do fato que há escassez de pesquisas, projetos e qualificação profissional. Dessa forma, no Amazonas, faz-se necessário pesquisas que eleve não apenas os parâmetros da região, mas também que deixe a sociedade, os pais, os autistas, e os profissionais mais alertas e cientes da real situação amazonense diante do TEA e o que se pode fazer para melhorá-la.

Diante disso, o presente estudo com o seu objetivo em identificação dos níveis de gravidade, determinação da incidência, da frequência e evidenciação das ações diagnosticas e problemáticas enfrentadas pelos pais, profissionais e autistas, é de grande importância para o futuro, visando a expansão cientifica e maior compressão e evolução acerca do transtorno do espectro do autismo.

\section{MÉTODOS}

Este trabalho de pesquisa realizado entre o período de Fevereiro de 2019 a Maio de 2020 caracteriza-se por apresentar caráter prospectivo, pela aplicação do teste Childhood Autism Rating Scale (CARS) com os autistas e questionário com os pais, e retrospectivo, através de prontuários com diagnóstico dos usuários da instituição de apoio, as quais serão mantidas em sigilo, em conformidade com o que prevê os termos de resolução no 466, de 12 de Dezembro de 2012 do Conselho Nacional de Saúde. A coleta dos dados somente foi realizada após a aprovação do Comitê de Ética em Pesquisa (CEP) número 3.129.189 e 3.593.876.

Nesta pesquisa foram incluídas todas as pessoas atendidas nas instituições de apoio pública que tinham diagnóstico do TEA confirmado por um médico. Os pais ou responsáveis das pessoas com TEA que participaram da pesquisa concordaram de forma voluntária e assinaram o Termo de Consentimento Livre e Esclarecido (TCLE). Os pais e/ou responsáveis das crianças com TEA não concordaram na utilização do Termo de Assentimento Livre e Esclarecido (TALE).

Os instrumentos empregados na pesquisa foram CARS, Anamnese Infantil, Questionário aos Pais, Questionário aos Psicólogos e Questionário Médico, como ferramentas de coleta de dados, contendo perguntas abertas. De acordo com RAPIN I e Goldman S (2008) o CARS tem uma escala de gravidade que 
pode ser déficit ausente, leve, moderado ou grave, e tem um escore diagnóstico de gravidade geral, dessa forma, a soma geral do escore da CARS pode variar entre um potencial de zero (sem características de autismo) a 60 (todas as características graves preenchidas). A Anamnese Infantil cada instituição tem o próprio questionário de acordo com as necessidades observadas.

O Questionário Médico foi desenvolvido com 8 perguntas abertas com a finalidade em saber sobre instrumento de diagnóstico e ideologias terapêuticas ao autista e a família, o Questionário aos Psicólogos tinha 9 perguntas sobre utilização de testes, tipo de terapia aplicada, tempo de trabalho com os autistas, maior desafio observado e o grau de satisfação, e o Questionário aos Pais tinha 6 perguntas abertas construídas pela equipe de pesquisa com perguntas pertinentes ao projeto como se há uso de medicação, idade da criança, idade do diagnostico, se estuda em escola regular ou excepcional, se há necessidade de mediador(a) e como a família lida e lidou com todo o processo do diagnóstico.

A pesquisa utilizou 3 categorias, na categoria (1) Pessoas do Transtorno de Espectro Autista envolveu observações no atendimento psicológico e na análise de questionários, numa amostra de 118 triagens referentes a usuários atendidos nas instituições. Na categoria (2) Responsáveis envolverá o Questionário aos pais, numa amostra de 114 triagens, e na categoria (3) Questionário aos profissionais numa amostra de 09 triagens cuja coleta de dados deu-se a partir de fevereiro de 2019.

Os riscos no desenvolvimento do estudo foram mínimos pois tratava-se de coleta de dados e entrevista através de ficha de dados pessoais, correspondendo a possíveis desconfortos psicológicos leves, entretanto poderia ocorrer algum constrangimento do participante mediante alguma pergunta relacionada aos seus hábitos. Nestes casos, foi respeitada a decisão de resposta do participante em não responder eventual pergunta.

Os benefícios que eram esperados com essa pesquisa era visualização da realidade do TEA em um município do Amazonas, a avaliação da qualidade de vida dos pacientes, o acompanhamento psicológico as famílias que necessitam identificar formas de equilibrar as relações familiares assim como ampliar o conhecimento para pais, profissionais e demais interessados e concluir como está a realidade amazonense em relação ao TEA.

Estabelecer parâmetros a fim de melhorar o tratamento dos pacientes diagnosticados com o TEA e indiretamente a contribuição para o avanço científico mostrar as doenças associadas que acometem as pessoas com TEA, para associá-las aos eventos clínicos do paciente, confirmar a hipótese diagnóstica feita pelo médico e explicar ao paciente e/ou responsáveis as suas alterações e peculiaridades. Assim, medidas como essas ajudam a perceber a evolução e o prognóstico da patologia, a frequência com que ela se manifesta nas instituições de apoio.

\section{RESULTADOS E DISCUSSÃO}

Os dados relativos as pessoas portadoras do TEA foram feitos através de observações no atendimento psicológico e análise de questionários no período de Fevereiro de 2019 a Maio de 2020. O CARS foi aplicado pelas psicólogas das instituições e as entrevistas com os pais pela aluna envolvida no projeto. Todas as pessoas com TEA tinham menos de 15 anos pois é a predominância das instituições envolvidas.

Em todas as instituições o que define a agilidade do atendimento é se tem disponibilidade de vagas. $\mathrm{Na}$ instituição 1 são atendidos de forma contínua cerca de 67 autistas, há atendimento com psicóloga, fonoaudióloga e educadora física, não é necessário encaminhamento para chegar a instituição e há uma fila de espera com cerca de 70 crianças, contudo a rotatividade é alta pela grande taxa de evasão além de existir uma politica de tolerância de 3 faltas.

$\mathrm{Na}$ instituição 2 no período da pesquisa tinha 1 autista, é um centro de apoio universitário dentro da universidade Nilton Lins, nele o atendimento é amplo, não sendo exclusivo a autistas, nele não é necessário encaminhamento e a lista de espera é pequena.

$\mathrm{Na}$ instituição 3 no período da pesquisa eram 11 autistas, o espaço oferece o serviço de psicologia e fonoaudiologia, e não há fila de espera. 
$\mathrm{Na}$ instituição 4 estavam cadastradas, no banco de dados pessoal da instituição, 3279 autistas. Existem 70 crianças em atendimento continuo com a psicologia. Cada médico atende em livre demanda cerca de 10 crianças por dia de atendimento. $\mathrm{O}$ atendimento é totalmente gratuito, contudo, é necessário encaminhamento de um médico para a instituição.

$\mathrm{Na}$ instituição 4 há um protocolo a ser seguido, aonde há uma ordem de consultas, primeiro os pais se encontram com o serviço social da instituição, posteriormente eles passam por uma consulta com a pediatra, caso ela veja que há necessidade é marcada uma consulta com o neuropediatra ou com o psiquiatra, nesta consulta entra a equipe multidisciplinar que a instituição oferece, aonde todas as pessoas que convivem com a criança passam juntas pela psicologia, além da criança passar também pela fonoaudiologia. Após o parecer dos profissionais da psicologia e fonoaudiologia é fechado o diagnóstico da criança pelo médico neuropediatra ou psiquiatra que a atendeu. A instituição conta com profissionais da área da odontologia, educação física, fonoaudiologia, assistência social, psicologia e medicina.

Rede de suporte social é uma interligação entre elementos sociais (família, serviços de saúde, instituições) que o indivíduo tem para enfrentar algum agravo, possibilitando o doente a ter condições melhores de vida (ROCHA G, et al, 2019).

Os dados foram relativos a 118 pessoas com diagnóstico de TEA, sendo que 107 eram do sexo masculino e 11 do sexo feminino, o que apesar de muitos estudos e teorias acerca da porcentagem maior de autismo em pessoas do sexo masculino ser bem clara, não há nada conclusivo (Gráfico 1). É natural que as mães queiram uma resposta de por que seu filho tem autismo; entretanto, o autismo ainda não possui uma etiologia claramente elucidada e comprovada, o que compromete a percepção das mães sobre este agravo (JÓZEFCZUK J, et al., 2017).

Gráfico 1 - Número de pessoas com TEA por gênero.

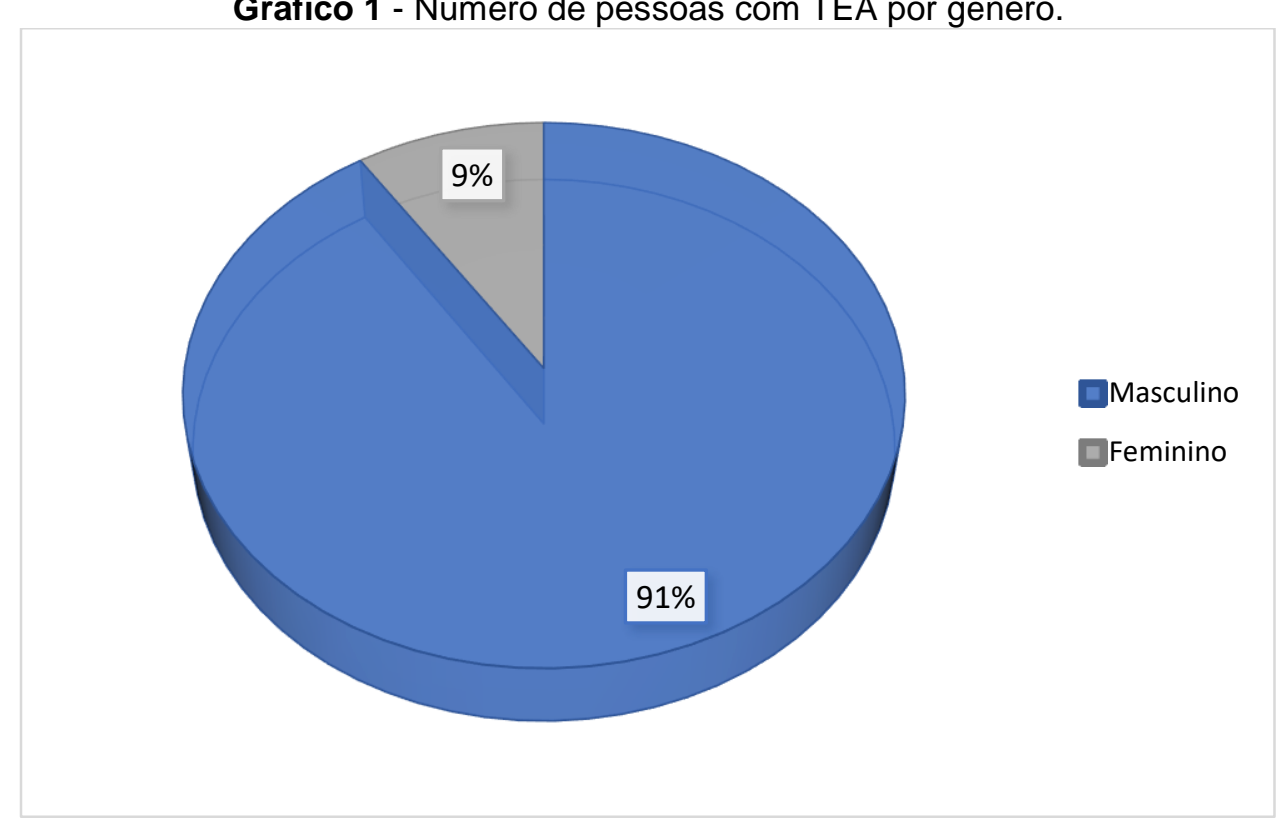

Fonte: Rolim FD, et al., 2021.

Concluiu-se, que 46 crianças possuíam nível severo, 19 moderado, 36 leve, sendo 4 Asperger e 13 não estavam dentro do TEA pelo teste CARS (Gráfico 2). Os resultados foram expostos e discutidos junto aos pais e responsáveis, o que ajudou a entendermos as dificuldades das pessoas com TEA e de seus pais e 0 reflexo disso no desenvolvimento do autista. A estruturação familiar mostrou-se de extrema importância na evolução das pessoas com TEA, desempenhando um papel igualitário as terapias, de forma que um desequilíbrio familiar, emocional ou na continuidade das terapias resulta em uma serie de regressões. Cada pessoa, tendo como familiar um autista ou não, tem suas opiniões sobre a origem do autismo e isso pode causar grande impacto sobre as emoções, atitudes e aceitação da situação (SILVA CM e NASCIMENTO HTB, 2017). 
Gráfico 2 - Porcentagem por nível de TEA.

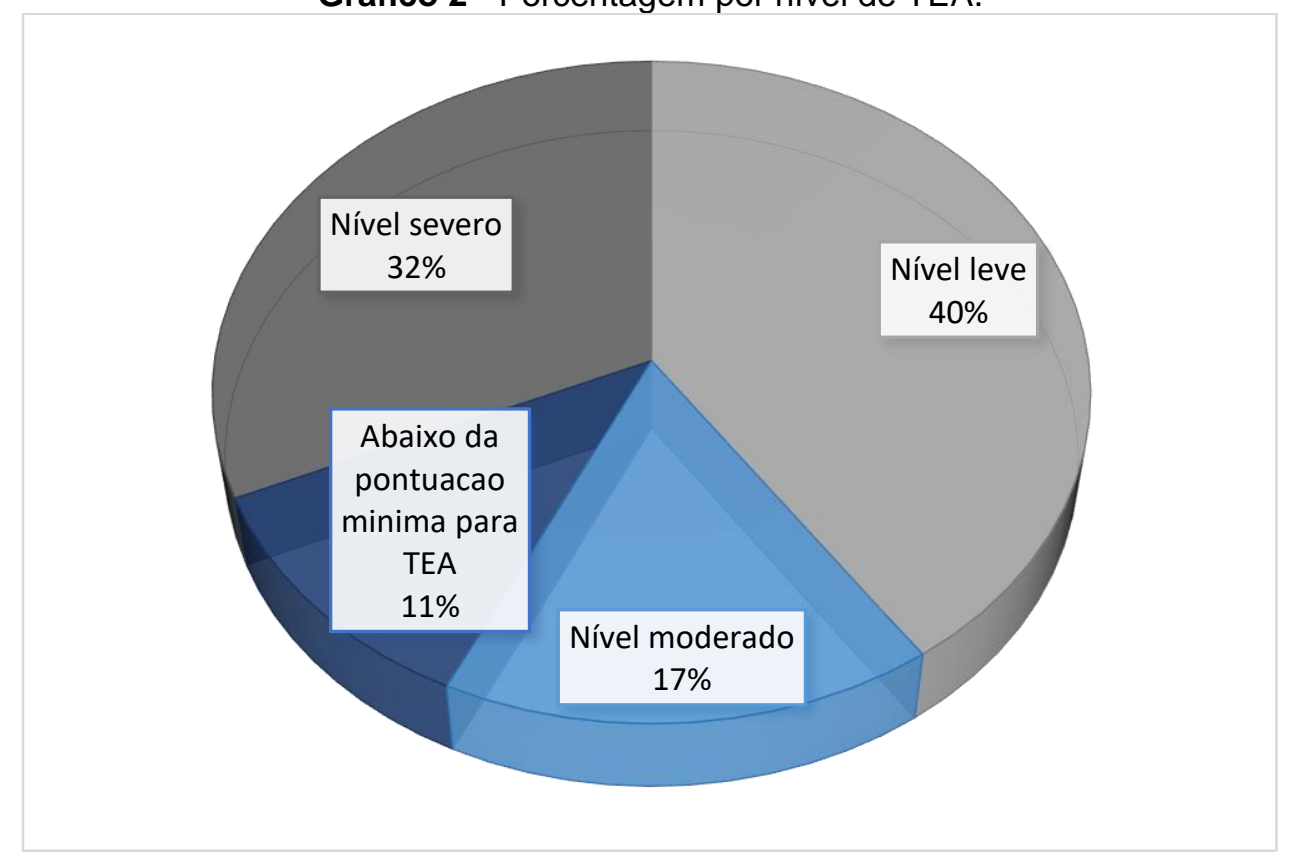

Fonte: Rolim FD, et al., 2021.

No entanto, vale ressaltar a problemática que em alguns casos leves aonde a pessoa com TEA regrediu muito no quadro autista, ao aplicar o teste CARS o resultado é abaixo da pontuação para TEA leve, nos fazendo refletir que as pessoas com autismo que tem tratamento contínuo e de qualidade podem regredir o quadro autista, podendo ser considerada não mais no mesmo nível de gravidade do inicio das suas terapias, dessa forma, frisando que, principalmente, a terapia psicológica e a consulta com um médico não podem ser abolidas, visto que o autismo não é uma doença com cura.

Levy SE e Hyman SL (2015) relatam que as terapias alternativas e complementares têm sido utilizadas para tratar sintomas de autismo em geral e sintomas comórbidos, como atenção, hiperatividade, irritabilidade, mau humor, sintomas gastrointestinais, convulsões, sono e sensibilidade tátil. Em alguns casos, as famílias selecionam as terapias alternativas quando as terapias convencionais parecem não afetar os sintomas centrais na tentativa de fornecer um tratamento mais abrangente. No questionário realizado com os pais, observou-se que $67,8 \%$ das crianças fazem uso de medicação de uso controlado. Destas os antipsicoticos (risperidona) e os psicoestimulantes (ritalina) são os mais utilizados. Em menor escala, porém também empregados tem os inibidores seletivos da recaptação de serotonina (Fluoxetina e Sertralina), estabilizadores de humor (Divalproato de Sódio) e os benzodiazepínicos (Clonazepam).

A alimentação é extremamente importante para qualquer ser vivo. Quando um ser vivo tem déficit nutricionais o seu corpo não desenvolve, não responde adequadamente, acarretando doenças, dessa forma, podemos fazer uma correlação clara entre pessoas sem autismo e pessoas portadoras do autismo, o autista com toda sua especificidade clínica, quando não quer comer determinados tipos de alimentos e/ou se sente mal ao comê-los tem manifestações exacerbadas, afinal, é difícil para ele falar e entender o que está tanto Ihe incomodando. Essa condição caracteriza-se pela pouca variedade de alimentos aceitos pela criança, dificultando a ingestão de alimentos com propriedades distintas, favorecendo, assim, a carência nutricional (FERREIRA M e SMEHA LN, 2018).

Assim foi visto que 53\% têm alguma alergia alimentar que consequentemente faz alterar seu comportamento e humor, se fazendo necessário um acompanhamento nutricional constante com profissionais adequados (nutricionista, médico e psicólogo), uma abordagem multiprofissional, para que se consiga contornar os efeitos de uma alergia ou rejeição alimentar (Gráfico 3). Crianças com TEA em sua maioria apresentam adversidades no processamento sensorial, dificuldades comportamentais, problemas gastrointestinais e fatores que estão relacionados com os alimentos como textura, sabor, cor, temperatura e consistência (PASTORINO V, et al., 2015). 
Gráfico 3 - Porcentagem de pessoas com TEA que possuem alguma alergia alimentar.

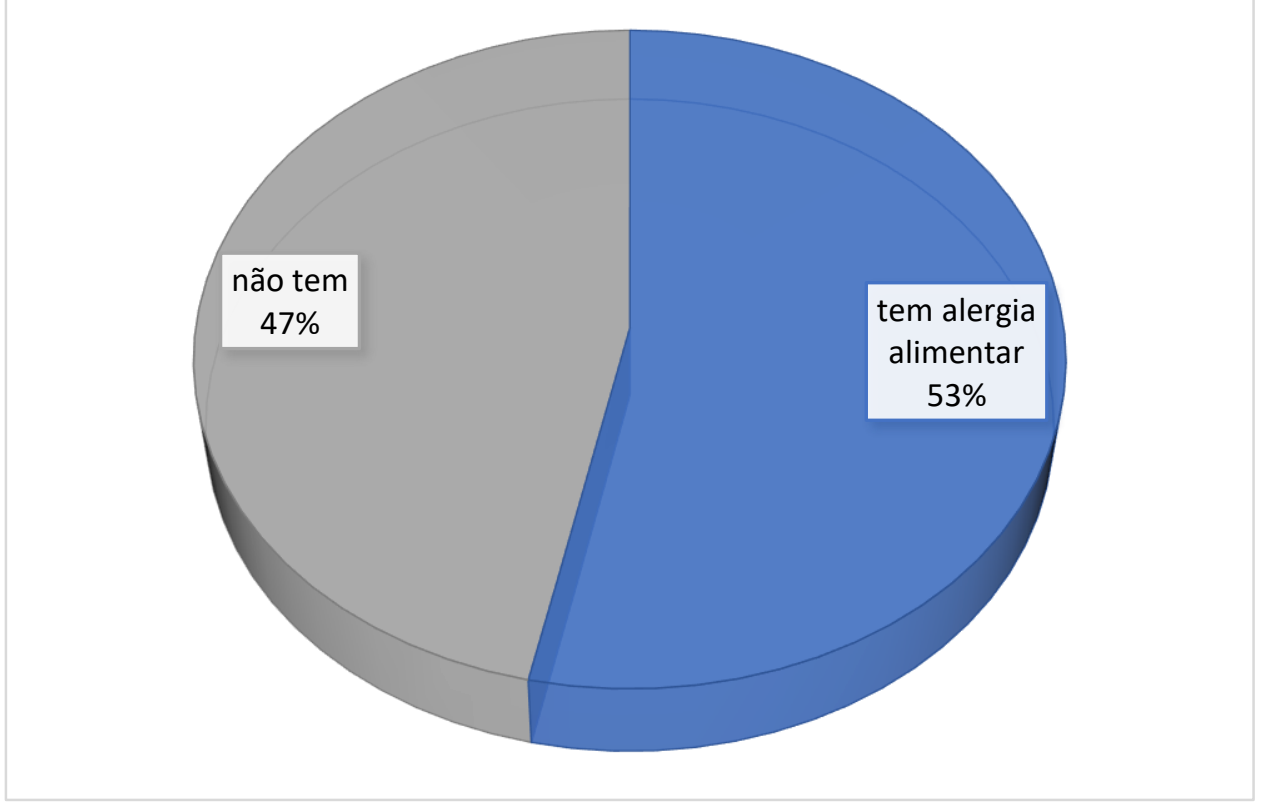

Fonte: Rolim FD, et al., 2021.

As pessoas com TEA infelizmente enfrentam diversos obstáculos na sua vida, o mundo não está preparado para elas que necessitam de um olhar diferenciado, uma abordagem mais atenciosa e adequada para que possam conseguir se desenvolver no âmbito escolar e social. Com isso, foi visto que $56,8 \%$ estão em escolas regulares e tem mediador, este é uma pessoa essencial para que ocorra uma conexão boa entre o autista, o educador e o assunto lecionado, necessário para que não se tenha evasão escolar e traumas, para que ele se sinta acolhido. No Brasil, o número de alunos com TEA matriculados em classes comuns ampliou $37,27 \%$ em um ano e embora as crianças sejam um ser humano em pleno desenvolvimento, verifica-se que aquelas em situação de TEA exibem uma maior incapacidade cognitiva, adversidades no relacionamento pessoal e na construção de vínculos, os quais são estabelecidos primeiramente com a família e com as instituições de educação, como creches e escolas (SILVA MM, et al., 2019).

Infelizmente para que se tenha um mediador muitas vezes os pais precisam lutar muito, várias vezes até juridicamente, exigir de forma incansável das escolas tanto particulares como publicas, por vezes tendo que ouvir a palavra "não" ou até "não estamos preparados para o seu filho". No Amazonas há poucas escolas excepcionais e a fila para conseguir uma vaga é grande, assim, apenas 6,7\% estão em escolas que são próprias para pessoas que necessitam de mais atenção.

Dos pais que foram entrevistados, todos relataram a dificuldade financeira e profissional em relação a médicos, terapias com psicólogo, fonoaudiólogo, educador físico, odontologista e nutricionista, além de mediador nas escolas com os filhos, o qual tem grande importância na relação professor e aluno, auxiliando no aprendizado e evolução do autista, alguns pais fazem o papel do mediador e ficam na escola durante o horário escolar para ajudar o professor em qualquer eventualidade que surja. Há, portanto, a necessidade de considerar neste contexto, as questões de ordem financeira e a vida de todos os membros do grupo familiar, não apenas o portador de Autismo e o seu tratamento. A exclusão social vivida pelas crianças autistas e percebidas pelos pais, causam profundo estresse emocional para aquela família, ao passo de se sentirem excluídas completamente da sociedade, uma vez que a criança é excluída, configurando um fator potencial de adoecimento mental (RIOS CC e KENNETH R, 2019).

No questionário realizado, com 4 psicólogos das instituições envolvidas, concluiu-se que os psicólogos não aplicam teste após o diagnóstico final do médico, a terapia cognitiva comportamental é a mais utilizada, e dentro dela há bifurcações nas quais cada psicólogo trabalha de acordo com a demanda da criança (Quadro 1). Além disso, foi visto que os maiores desafios observados por eles é o estresse pelos sinais e sintomas, a 
falta de apoio, a demora no fechamento do diagnóstico e principalmente a não elaboração do luto por parte dos pais pelo fato de haver um planejamento e idealização em cima de um filho. Percebe-se como essas mães vivenciam uma perda de expectativas depositadas na criança e quando a criança nasce com alguma limitação significante, a frustação compromete a percepção da mãe para com aquele filho (FERREIRA $M$, et al., 2018).

Dessa forma, há a não compreensão que na realidade a criança é perfeita do jeito dela, os pais não conseguem e não sabem lidar com a nova realidade, onde seus sonhos são desfeitos. Por fim, o grau de satisfação dos psicólogos é positivo, segundo eles, estão na área por amor e pediram para ir trabalhar com autistas.

Quadro 1 - Questionário aos psicólogos.

\begin{tabular}{|ll|}
\hline 1. & Qual a abordagem inicial? \\
\hline 2. & Aplica algum teste? \\
\hline & 2.1. $\quad$ Se sim, qual? \\
\hline & 2.2. $\quad$ Após o teste, qual é o próximo passo? \\
\hline 3. & Como você escolhe a terapia adequada? \\
\hline 4. & Há quantos anos trabalha com autistas? \\
\hline 5. & Como entrou nessa área? \\
\hline 6. & Qual linha se identifica mais? \\
\hline 7. & Qual a faixa etária que trabalha? \\
\hline 8. & Maior desafio observado. \\
\hline 9. & Grau de satisfação. \\
\hline
\end{tabular}

Fonte: Rolim FD, et al., 2021.

Em suma, foi realizado o questionário médico com 4 médicos que trabalham com autistas, assim, na forma de diagnóstico pode-se vê que todos os médicos fazem uso do instrumento de diagnóstico da sua preferência, podendo ser CARS, Avaliação de Traços Autísticos (ATA) ou Questionário Modificado para a Triagem do Autismo (M-CHAT), entre outros (Quadro 2).

Quadro 2 - Questionário ao Médico.

\begin{tabular}{|c|c|}
\hline \multicolumn{2}{|c|}{ 1. Usa instrumento de diagnóstico? } \\
\hline 1.1. & Qual é o instrumento de diagnóstico? \\
\hline 1.2. & Caso NÃO use, como é feito o diagnóstico? \\
\hline \multicolumn{2}{|c|}{ 2. Faz anamnese antes? } \\
\hline 2.1. & O que compõe a anamnese? \\
\hline 3. Com & a linha de suspeita, raciocínio? \\
\hline 4. Fazu & do auxílio psicológico para o diagnóstico? \\
\hline \multicolumn{2}{|c|}{ 5. Faz uso de exames complementares? } \\
\hline 5.1. & Quais? \\
\hline \multicolumn{2}{|c|}{ 6. Quais terapias utiliza? } \\
\hline 6.1. & Faz uso de terapêutica medicamentosa? \\
\hline 6.2. & Tenta algum mecanismo antes de prescrever a terapia medicamentosa? \\
\hline 6.3. & Tenta retirar a terapia medicamentosa? \\
\hline 6.4 . & Faz controle do uso da terapia medicamentosa? \\
\hline \multicolumn{2}{|c|}{ 7. Conhece a terapia ortomolecular? } \\
\hline 7.1 & Se sim, o que pensa sobre? \\
\hline \multicolumn{2}{|c|}{ 8. Encaminha a criança a algum outro especialista? } \\
\hline 8.1. & Faz uso da equipe multidisciplinar? \\
\hline 8.2. & Recomenda as instituições de apoio? \\
\hline 8.3. & Recomenda aos pais a terapia psicológica? \\
\hline 8.4. & Faz abordagem psicológica? \\
\hline
\end{tabular}

Fonte: Rolim FD, et al., 2021. 
Foi observado que as queixas dos pais são ouvidas, que é feito uso de exames complementares, quando se acha necessário, como auditivos, ressonância do crânio, eletroencefalograma, exames de triagem metabólica, além de se fazer o diagnóstico de exclusão junto a uma equipe multidisciplinar como profissional da psicologia, da fonoaudiologia, médico otorrinolaringologista.

As terapêuticas utilizadas são diversas, englobam terapia psicológica e fonoaudiologica que dentro do possível é incluído os pais, foi observado que há uso da terapia ortomolecular aonde é usado a linha de raciocínio de suplementação nutricional e reposição de vitaminas, pelo fato de haver muitas vezes uma restrição ou rejeição alimentar por parte do autista, terapia homeopática e por fim, foi visto que a terapia medicamentosa é a mais utilizada e muitas vezes a principal para ajudar os autistas e pais. Dessa forma, observou-se que cada médico tem suas crenças e linha de raciocínio, influenciando na conduta.

\section{CONCLUSÃO}

O presente estudo contribuiu para a identificação de pessoas que não se adequavam ao espectro do autismo, mas tinham diagnóstico de TEA, assim como a identificação dos níveis e frequência da gravidade que foram confirmadas ao olhar dos pais e profissionais. A estruturação familiar mostrou-se de extrema importância na evolução das pessoas com TEA, desempenhando um papel igualitário as terapias. Uma pessoa com autismo que tem um acompanhamento multidisciplinar onde há pais e profissionais juntos a favor, se beneficia de forma extraordinária. Foi visto como problemática o psicológico dos pais, o financeiro, a falta de profissionais de qualidade, de profissionalização e a falta de conexão entre os profissionais envolvidos. $O$ diagnóstico médico e os terapeutas evoluíram muito, tendo cada dia mais profissionais capacitados, porém existe uma barreira financeira e de vagas em locais especializados que aclama por atenção.

\section{AGRADECIMENTOS E FINANCIAMENTO}

Primordialmente agradecemos aos pais e responsáveis. Por conseguinte, agradecemos a todos os profissionais que deram apoio a aluna, o seu orientador, aos psicólogos e médicos engajados em ajudar e contribuir com esse projeto. Por fim, agradecemos à Universidade Nilton Lins que forneceu bolsa de iniciação científica a aluna.

\section{REFERÊNCIAS}

1. DIAGNOSTIC AND STATISTICAL MANUAL OF MENTAL DISORDERS (DSM-5). Diagnostic and Statistical Manual of Mental Disorders.American Psychiatric Association, 2013; 50-59.

2. ALMEDA CM, ALBUQUERQUE K. Autismo: Importância da Detecção e Intervenção Precoces. Revista Científica Multidisciplinar Núcleo do Conhecimento. 2017; 1: 488-502.

3. BRASIL. Linha de cuidado para a atenção às pessoas com transtornos do espectro do autismo e suas famílias na Rede de Atenção Psicossocial do Sistema Único de Saúde. Secretaria de Atenção à Saúde, Departamento de Atenção Especializada e Temática. - Brasília: Ministério da Saúde, 2015.

4. CAMPOS CC, et al. Expectativa de profissionais da saúde e de psicopedagogos sobre aprendizagem e inclusão escolar de indivíduos com transtorno do espectro autista. Revista Psicopedagogia. 2018; 35(106): 3-13.

5. CARMO MA, et al. O ambiente familiar e o desenvolvimento da criança com autismo. Rev enferm UFPE online. 2019; 13(1): 206-15.

6. FERNANDES A, et al. A criança com transtorno do espectro autista (TEA). Revista de Terapia Ocupacional da Universidade de São Paulo. 2018; 29: 187-194.

7. FERREIRA MM, FRANÇA AP. O Autismo e as Dificuldades no Processo de Aprendizagem Escolar. Id online Rev. Mult. Psic. 2017; 11(38): 507-519.

8. FERREIRA M, SMEHA LN. A experiência de ser mãe de um filho com autismo no contexto da monoparentalidade. Psicologia em Revista. 2018; 24(2): 462-481.

9. FURTADO L, et al. Transtornos globais de desenvolvimento e saúde pública: uma revisão integrativa. Rev. Percurso Acadêmico, 2014; 4: 283-297.

10. García BL, Planells E, Pozo PP, López JM. Association Between Feeding Problems and Oral Health Status in Children with Autism Spectrum Disorder. J AutismDevDisord. 2019,49: 4997-5008.

11. GOMES PTM, et al. Autismo no Brasil, desafios familiares e estratégias de superação: revisão sistemática. J. Pediatr. 2015; 91(2): 111-121. 
12. JÓZEFCZUK J, et al. Homocysteine as a Diagnostic and Etiopathogenic Factor in Children with Autism Spectrum Disorder. Journalof Medicinal Food. 2017; 20(8).

13. LEVY SE, HYMAN SL. Complementary and alternative medicine treatments for children with autism spectrum disorders. Child Adolesc Psychiatr Clin N Am. 2015; 24(1): 117-143.

14. NEVES AT, et al. O processo de inclusão de crianças com necessidades especiais de saúde na educação. Ver Enferm UFSM. 2017; 7(3): 374-387.

15. Padmanabhan PS e Shroff $\mathrm{H}$. The relationship between sensory integration challenges and the dietary intake and nutritional status of children with Autism Spectrum Disorders in Mumbai. India. International Journal of Developmental Disabilities. 2018; 66(2): 142-152.

16. PASTORINO V, et al. Clinical differences in children with autism spectrum disorder waith and without food selectivity. Roma. 2015; 1-6.

17. PEREIRA JRS, et al. AUTISMO: LIDANDO COM AS DIFICULDADES E PESPECTIVAS DO CUIDADO. Caderno De Graduação - Ciências Biológicas E Da Saúde - UNIT - SERGIPE. 2021; 6(3), 33.

18. Pinto RNM, et al. Autismo infantil: impacto do diagnóstico e repercussões nas relações familiares. Rev Gaúcha Enferm. 2016; 37(3): e61572.

19. RAPIN I, GOLDMAN S. A escala CARS brasileira: uma ferramenta de triagem padronizada para o autismo. J. Pediatria. 2008; 84(6): 473-475.

20. RIESGO R, et al. Transtornos da aprendizagem: abordagem neurobiológica e multidisciplinar. 2 ed. Brasil: Artmed, $2015 ; 512$.

21. RIOS CC, KENNETH R. Especialismo, especificidade e identidade - as controvérsias em torno do autismo no SUS. Ciência \& Saúde Coletiva. 2019; 24(3): 1111-1120.

22. ROCHA G, et al. Análise da seletividade alimentar de crianças com transtorno do espectro autista. Electronic jornal Collection Health, 2019; 24: 538, 1-8.

23. SILVA MM, et al. A Inclusão Educacional de Alunos com Autismo: Desafios e Possibilidades. Id online Rev. Mult. Psic. 2019; 13(43): 151-163.

24. SILVA CM, NASCIMENTO HTB. Perturbações do Espectro do Autismo: Uma Revisão Bibliográfica dos Benefícios da Prática de Educação Física em Alunos com Necessidades Educativas Especiais. 2017; 2: 1-14.

25. SOUSA PML, SANTOS IMSC. Caracterização da Síndrome Autista. Psicologia.pt. Portal dos Psicólogos. 2018.

26. ZANON RB, et al. Identificação dos Primeiros Sintomas do Autismo pelos Pais. Psicologia: Teoria e Pesquisa. 2014; $30(1)$ : 25-33. 